\title{
Majority Decision Functions of up to Six Variables
}

\author{
By S. Muroga, I. Toda, and M. Kondo
}

1. Introduction. Recently logical elements based essentially on the majority decision principle have been widely used in electronic computers. Among these elements are parametrons, magnetic cores, transistor-resistor logic, et cetera.

The logical behavior of such elements can be expressed by a model called a "majority decision element" with $n$ Boolean inputs and one Boolean output, whose operation can be described in the form of a logical function called a "majority decision function".

This paper defines the canonical representative of each equivalence class in the classification of the majority decision functions by complementing and permuting variables and by complementing the output. Also, a method is proposed to obtain all the representatives with their optimum structures, and a table of the representatives of the majority decision functions of up to six variables is provided.

The reader should be familiar with the content of a previous paper by the authors, included as reference [1].

2. Majority Decision Functions. A "majority decision element" of $n$ variables is a logical element with $n$ Boolean inputs, $x_{1}, x_{2}, \cdots, x_{n}$ and one Boolean output. The output value of the element is

$$
\begin{array}{ll}
\text { one for } & \sum_{i=1}^{n} w_{i} x_{i} \geqq T \\
\text { zero for } \quad & \sum_{i=1}^{n} w_{i} x_{i} \leqq T-1
\end{array}
$$

where $w_{i}$ is a prescribed constant real number called a "coupling weight" associated with the input $x_{i}$ and $T$ is also a prescribed constant real number called a "threshold."

In the case of parametrons or magnetic cores, the coupling weight $w_{i}$ corresponds to the number of turns of the winding of the input $x_{i}$. The threshold $T$ is related to the number of turns $w_{c}$ for the constant input by the relation,

$$
w_{c}=\sum_{i=1}^{n} w_{i}+1-2 T
$$

where $w_{c}>0$ means the constant of one is coupled to the element and $w_{c}<0$ means the constant of zero.

A set of $(n+1)$ real numbers $\left(w_{1}, w_{2}, \cdots, w_{n} ; T\right)$, which specifies the behavior of a majority decision element, will be called a "structure" of the element.

A logical function represented by a single majority decision element will be called a "majority decision function."

Received September 22, 1961.

* The term -1 on the right hand side is introduced as a normalizing factor of $w_{i}$ 's and $T$. 
For example, a majority decision element with the structure $(2,1,1 ; 2)$ represents a function $x_{1}+x_{2} x_{3}$; hence, this function is a majority decision function. In contrast, the function $x_{1} x_{2}+x_{3} x_{4}$ is not a majority decision function since it can not be realized by any single majority decision element.

3. Classification of Majority Decision Functions. Logical functions obtained from a given logical function $f$ by the following operations are defined as equivalent functions with $f$ :

(1) Complementation of one or more input variables,

(2) Permutation among input variables,

(3) Complementation of $f$.

It is a well known fact that the logical functions can be classified into equivalent classes by this equivalent relation. Once a structure of a majority decision function is given, its equivalent functions can be easily realized in the same element by complementing and/or permuting input variables and/or by complementing the output. Thus, it is not necessary to determine the whole of the majority decision functions; it is sufficient to know the representatives of their equivalence classes. It should be noted that this limits the study to a much smaller number of functions.

In the case of general logical functions, it is difficult to extract systematically one representative from each equivalence class, but in the case of majority decision functions there is a way to define a canonical representative of each equivalence class from the intrinsic nature of majority decision functions.

The method of determining the canonical representative is described below. Hereafter in this section the majority decision function is assumed to have $n$ nonvacuous variables.

Any majority decision function can be expressed by a polynomial without any complemented variable by appropriately complementing one or more variables (refer to [1], Section 3). Such a polynomial will be called a "positive polynomial." The way to complement the variables to obtain a positive polynomial from a given function is unique if complementing one variable more than once is prohibited. Hence we can restrict the possible representatives within positive polynomials. This is equivalent to the condition in which the representative should be realized by a majority decision element with positive coupling weights.

All the variables of a majority decision function can be ordered by a relation $\succsim$ (refer to [1], Definition 3 and Theorem 1). Therefore, it is always possible for variables to be permuted and relabelled so that $x_{1} \succsim x_{2} \succsim \cdots \gtrsim x_{m}$ holds. This permutation can be uniquely determined except in the case of arbitrary permutations among some variables such as $x_{1}, x_{2}, \cdots, x_{m}$ for which $x_{1} \sim x_{2} \sim \cdots \sim x_{m}$ holds. But $x_{1} \sim x_{2} \sim \cdots \sim x_{m}$ means that the given function is symmetric with respect to these variables, and therefore the function is invariant under the permutations among $x_{1}, x_{2}, \cdots, x_{m}$. Thus, the function for which $x_{1} \succsim x_{2} \succsim \cdots \succsim x_{n}$ holds is unique and can well be adopted as a possible representative. Of course, this is equivalent to the condition in which $w_{1} \geqq w_{2} \geqq \cdots \geqq w_{n}$ holds for the representative majority decision element. Note that as a conclusion from the above requirements, we have $w_{1} \geqq w_{2} \geqq \cdots \geqq w_{n}>0$ except $w_{c} \leqq 0$.

Only two functions left in each class satisfy both of the conditions just described. 
If we denote one of them by $f$, the other is the dual function $f^{*}$ of $f$. But for a majority decision function, either $f^{*} \supseteq f$, or $f \supseteq f^{*}$ holds (refer to [1], Corollary 2). A unique representative of the equivalent class can be determined by requiring either of the two inequalities. If we adopt $f$ such that $f \subseteq f^{*}$, this implies $w_{c} \leqq 0$.

Thus, it is shown that there is a unique canonical representative in each equivalent class of majority decision functions which satisfies the following three conditions:

Conditions $I$.

(1) A positive polynomial,

(2) $x_{1} \succsim x_{2} \succsim \cdots \succsim x_{n}$

(3) $f$ such that $f \subseteq f^{*}$.

Given a majority decision function, we can now effectively obtain the representative of the equivalent class to which the given function belongs.

4. A Method to Obtain the Totality of the Representatives of the Majority Decision Functions. From Section 5 of [1] it can be determined by linear programming whether a given function is a majority decision function or not. Therefore, it is possible, at least in principle, to obtain the totality of majority decision functions by applying the criterion to all of $2^{2^{n}}$ logical functions of $n$ variables. It will, however, take an impractically long time to solve $2^{2^{n}}$ linear programming problems for large values of $n$, but the length of time to perform computation will be greatly reduced if we can confine the scope of the functions to be tested.

Accordingly, a method is developed here to obtain a set of logical functions which includes all the representatives of majority decision functions and to apply the criterion only to those functions in the set. The functions in the set will be called "candidates" of the representatives.

Any positive majority decision function can be expressed in the form of $M x_{1}+$ $N$, where $M$ and $N$ are both positive majority decision functions of $(n-1)$ variables, $x_{2}, x_{3}, \cdots, x_{n}$. Therefore, without loss of generality, we can restrict the candidates within such functions. This assumes that we have already obtained all the majority decision functions of $(n-1)$ variables; hence the method described here is one of the recursive constructions of majority decision functions with respect to the number of variables.

Moreover, if we choose as the candidates those functions for which Conditions I can be defined, then the set of the candidates will certainly contain the totality of the representatives of the majority decision functions of $n$ variables.

Then the restrictions imposed upon combinations of $M$ and $N$ will be examined.

Condition (1) will be trivially satisfied, for $M x_{1}+N$ is positive from its construction.

Condition (2) requires that the relation

$$
x_{2} \succsim x_{3} \succsim \cdots \gtrsim x_{n}
$$

must hold for both $M$ and $N$. Moreover, in order that $x_{1} \succsim x_{2}$ may hold in $M x_{1}+$ $N$, it is necessary (Corollary 1 of Reference [1]), that

$$
m_{2} \supseteq n_{1},
$$


where

$$
\begin{aligned}
& m_{2}=M\left(0, x_{3}, \cdots, x_{n}\right) \\
& n_{1}=N\left(1, x_{3}, \cdots, x_{n}\right) .
\end{aligned}
$$

As the relation $\succsim$ is an ordering relation (Theorem 1 of [1]), the relation

$$
x_{1} \succsim x_{2} \succsim \cdots \succsim x_{n}
$$

follows from (3) and (4).

$M$ and $N$ are majority decision functions satisfying (3), hence the relations

$$
m_{1} \supseteq m_{2} \quad \text { and } \quad n_{1} \supseteq n_{2}
$$

where

$$
\begin{aligned}
& m_{1}=M\left(1, x_{3}, \cdots, x_{n}\right) \\
& n_{2}=N\left(0, x_{3}, \cdots, x_{n}\right)
\end{aligned}
$$

hold (Corollary 1 of Reference [1]). From (4) and (6) we have

$$
M \supset N \text {. }
$$

From (3) in Conditions I, it is necessary that

$$
f^{*}=N^{*} x_{1}+M^{*} N^{*} \supseteq f=M x_{1}+N .
$$

But as $M^{*} N^{*}=M^{*}$ from (7), (8) reduces to

$$
M^{*} \supseteq N \text {. }
$$

Thus, we choose as candidates those functions which satisfy the following conditions:

\section{Conditions II}

(1) Both $M$ and $N$ are positive majority decision functions of $(n-1)$ variables, $x_{2}, x_{3}, \cdots, x_{n}$.

(2) For both $M$ and $N, x_{2} \succsim x_{3} \succsim \cdots \gtrsim x_{n}$.

(3) $m_{2} \supseteq n_{1}$.

(4) $M^{*} \supseteq N$.

By taking all the combinations of $M$ and $N$ which satisfy Conditions II, we can obtain the set of candidates of the representatives of majority decision functions of $n$ variables.

$M$ and $N$ must satisfy (1) and (2) of Conditions II. Such functions are either canonical representatives of majority decision functions or their dual functions. Therefore, once the totality of representatives of majority decision functions of $(n-1)$ variables are obtained, the scope within which functions $M$ and $N$ must be taken can be easily determined. In this way we can obtain the totality of the representatives of majority decision functions of $n$ variables recursively.

The next problem is to examine each candidate to determine whether or not it is a majority decision function. If so, it is clearly a canonical representative of an equivalent class defined in the preceding section. The discrimination of majority decision functions from other functions can be accomplished by linear programming. The details will be found in Section 5 of [1]. 
5. Majority Decision Functions of up to Six Variables. Following the procedure described in Section 4, a program was written for the parametron digital computer MUSASINO-I, and all the canonical representatives of the functions of up to six variables were obtained.

The canonical representatives of up to five variables had been obtained by $\mathrm{S}$. Muroga [3] at that time, using a combinatorial method. Both results agreed completely.

The canonical representatives of the functions of up to six variables are shown in Table 1. The functions are numbered according to the magnitude of $V=\sum_{i=1}^{n} w_{i}$, which is expected to denote the complexity of functions to some extent. Functions are expressed by denoting the variables by means of their subscripts. For instance, $12+13+23$ stands for the function $x_{1} x_{2}+x_{1} x_{3}+x_{2} x_{3}$.

In the same entry of the table an optimum structure of the function is shown. The optimum structure is one with a minimum number of total turns of windings, namely, a structure which minimizes $\left(w_{1}+w_{2}+\cdots+w_{n}+\left|w_{c}\right|\right.$ ) (Section 5 in [1]).

To establish the threshold $T$, the constant input of zero must be coupled to the element with a winding of $2 T-V-1$ turns. Dual functions can be realized by merely reversing the polarity of the constant input, that is, by coupling the constant of one to the same winding.

The numbers in this table are somewhat different from those shown in [1]. This is because $f$ and $f^{*}$ are considered to belong to the same equivalence class in this paper and that in Table 1 the numbers of functions of $n$ (nonvacuous) variables are shown, while the numbers for up to $n$ variables are shown in [1].

By computing the number of the members of each equivalent class, the total numbers of majority decision functions are obtained and shown in Table 2.

6. Remarks on the Results. Some remarks are added here concerning the representatives of majority decision functions of up to six variables.

First, it is remarkable that all the candidates proved to be true representatives, that is, Conditions II are sufficient for a function of up to six variables to be realized by a single majority decision element.

Second, it is interesting to note that the optimum structures $\left(w_{1}, w_{2}, \cdots, w_{n}\right)$ are all integer-valued in spite of the fact that the optimum structure is obtained as a solution of a system of inequalities of the form of equation (1).

A structure of a majority decision function is a solution of a system of $2^{n}$ linear inequalities (Section 5 of Reference [1]).

$$
\begin{aligned}
A x \geqq b \quad A & =\left\{\begin{array}{c}
a_{i j} i \downarrow 1,2, \cdots, 2^{n} \\
i \rightarrow 1,2, \cdots, n
\end{array}\right\} \\
x & =\left[\begin{array}{c}
w_{1} \\
w_{2} \\
\vdots \\
w_{n} \\
T
\end{array}\right]
\end{aligned}
$$

The third remark concerns the structure of the solution space of these inequalities. It has been noted that for a majority decision function of up to five 


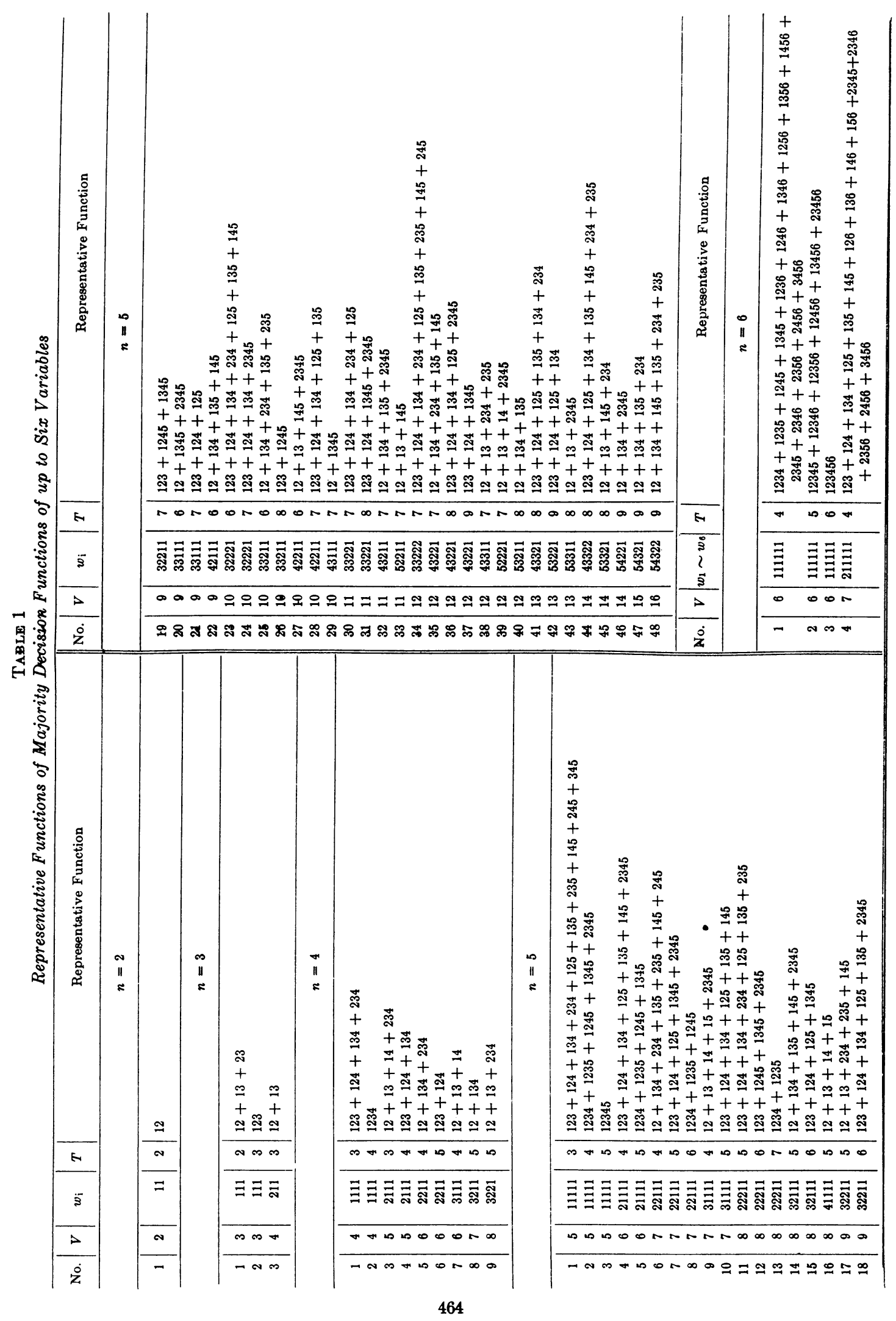




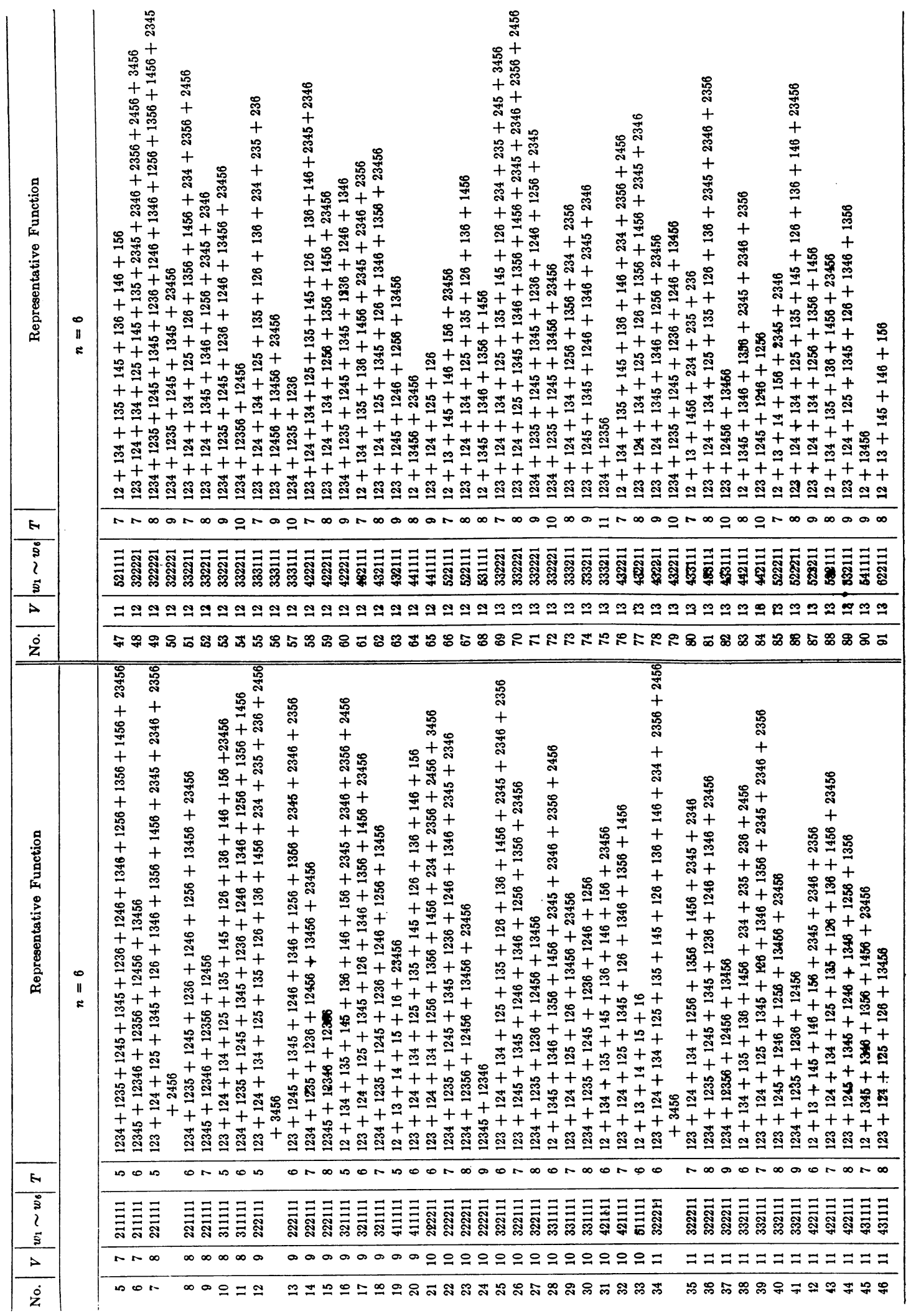




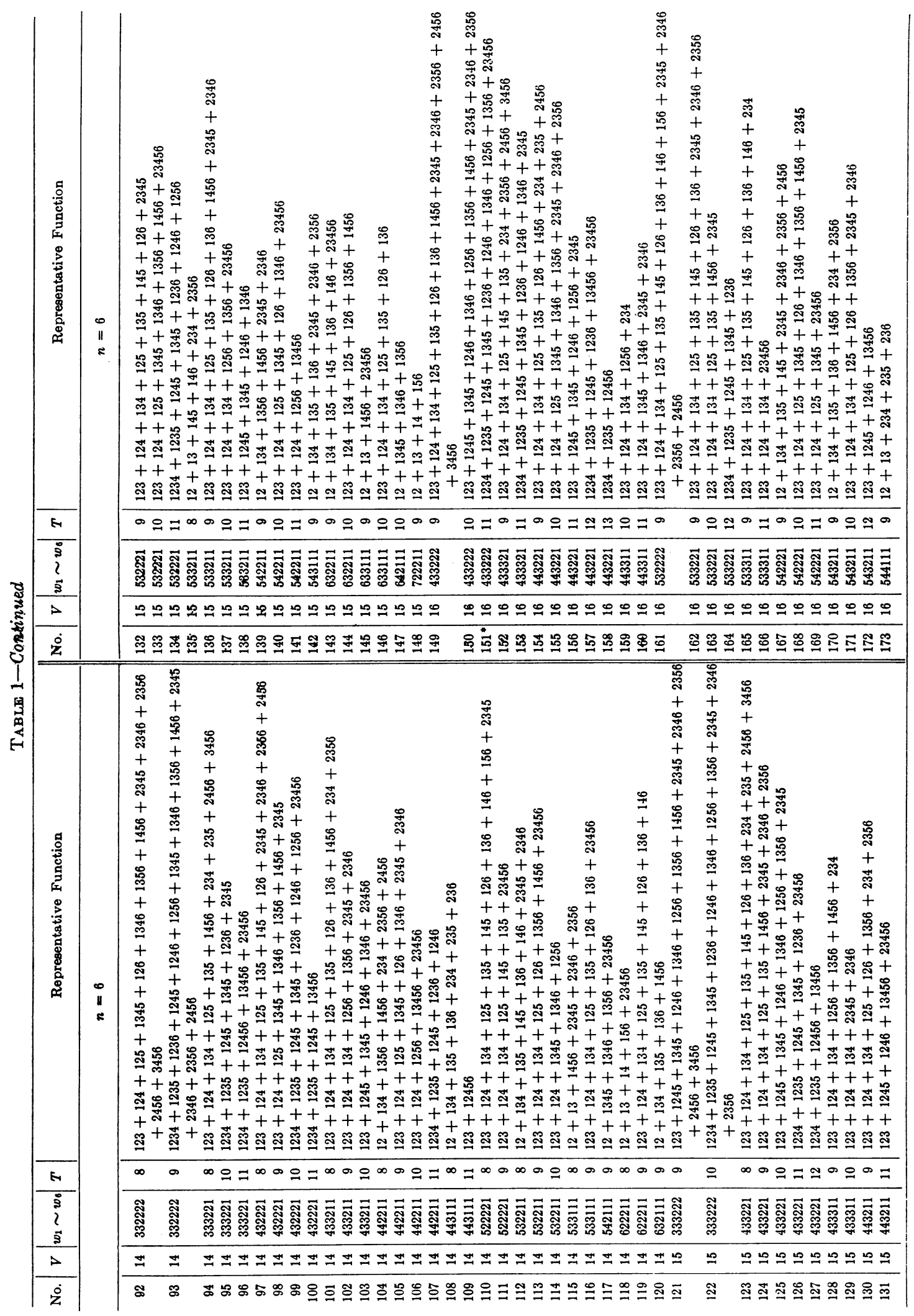




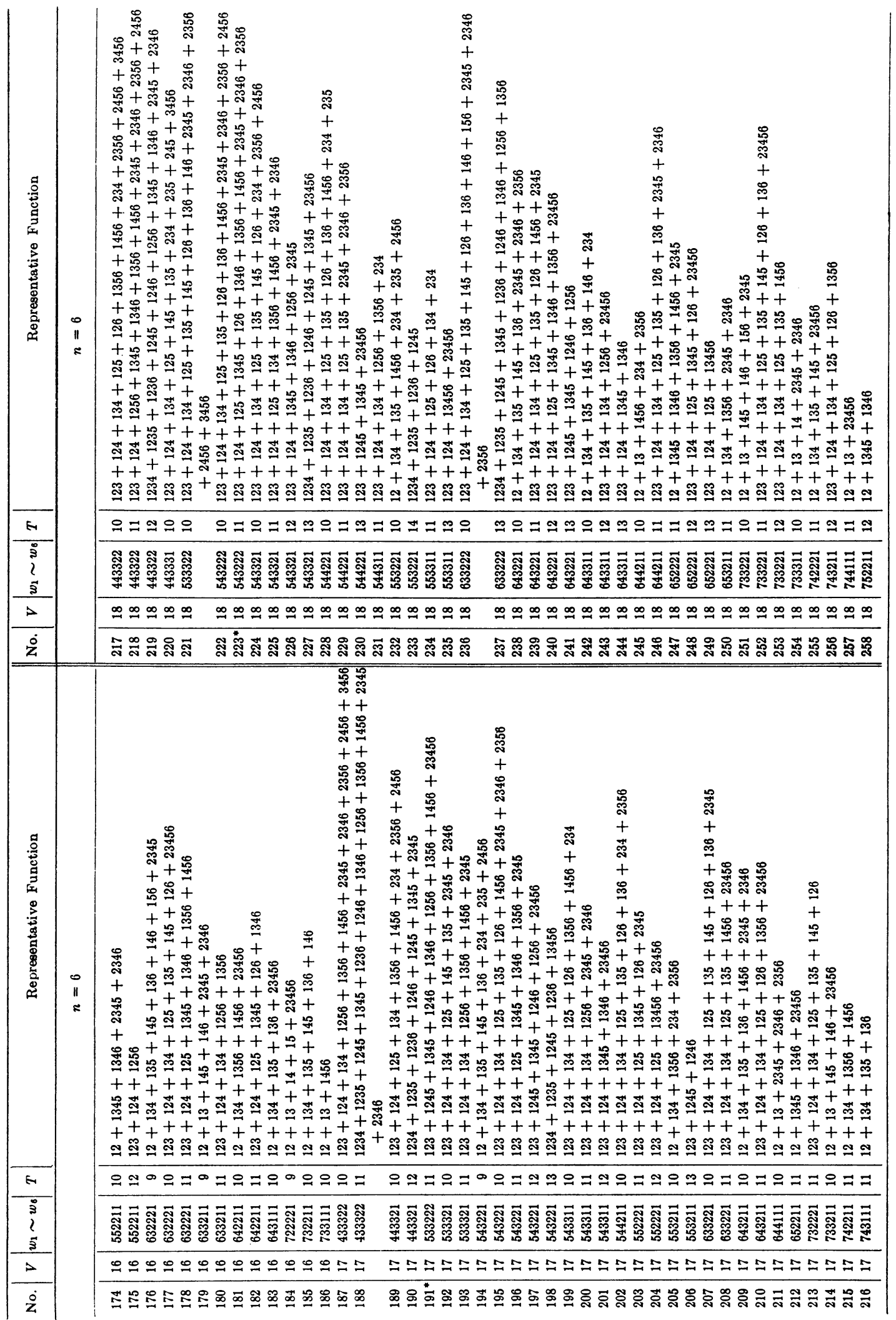




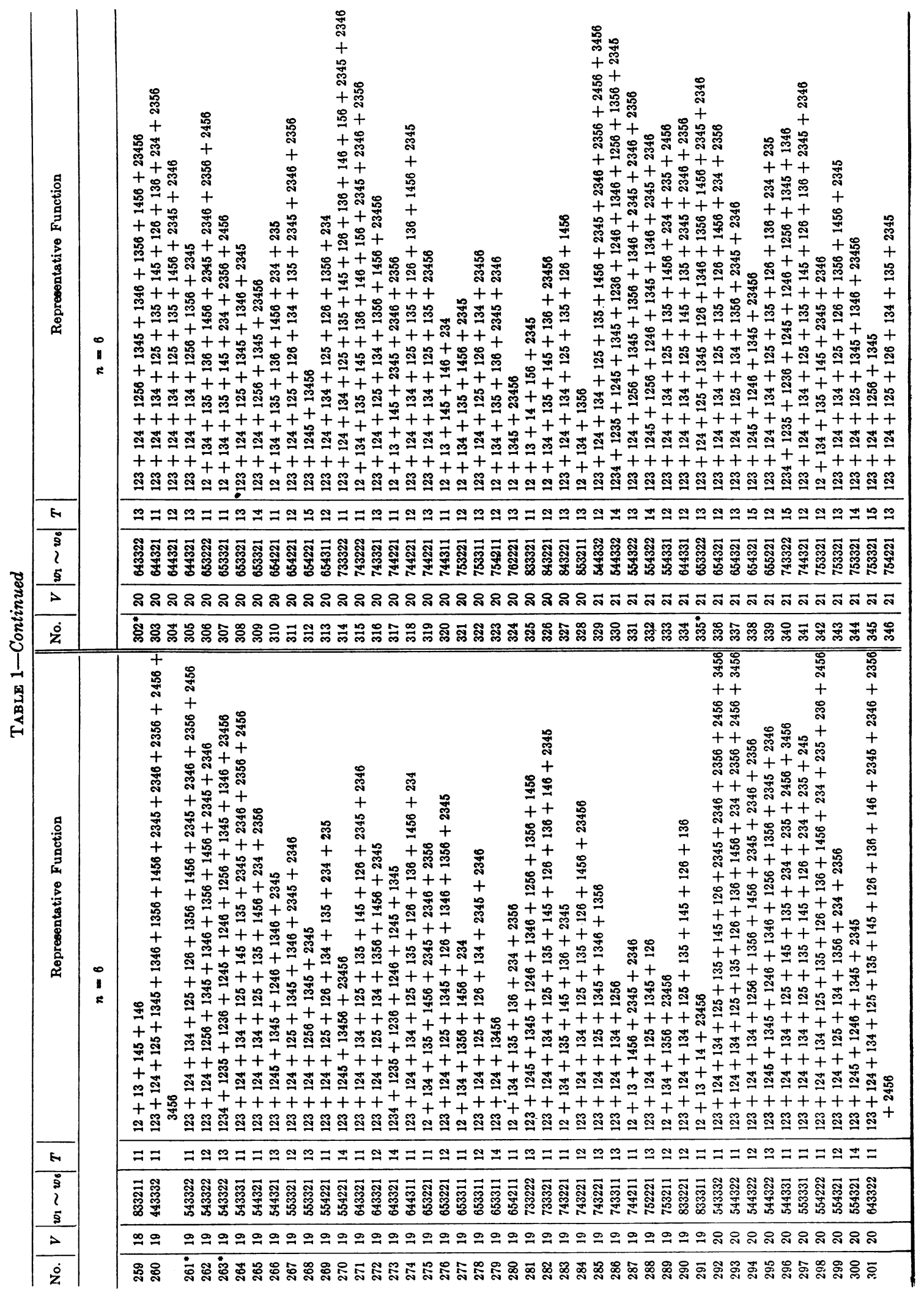




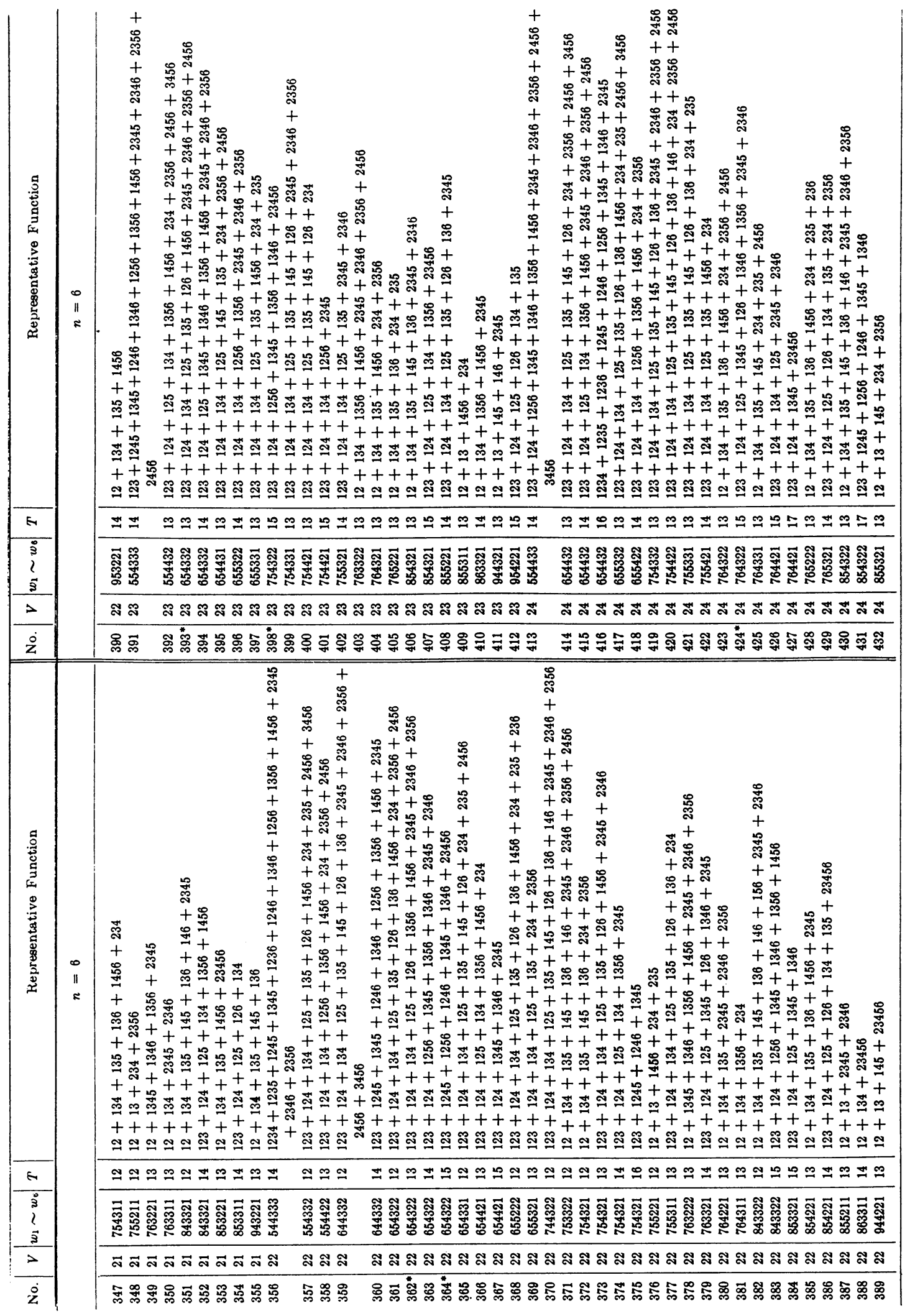




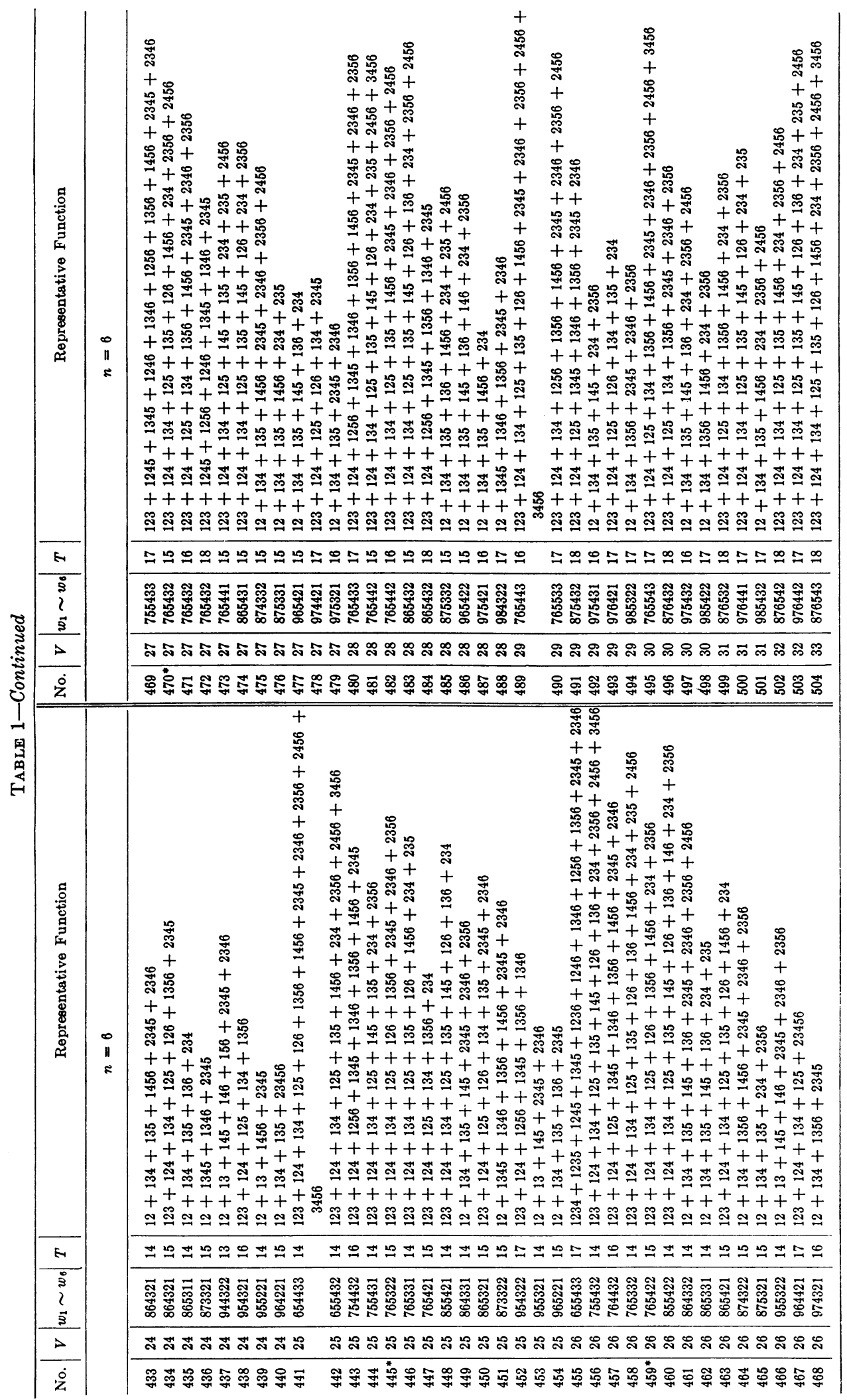


TABLE 2

The Number of Majority Decision Functions

\begin{tabular}{r|r|r|r|r|r}
\hline$n$ & $\begin{array}{c}\text { Number of Logical Functions } \\
\text { of up to } n \text { Variables }\end{array}$ & $\begin{array}{c}\text { Number of Types } \\
\text { of Logical } \\
\text { Functions } n \\
\text { Variables* }\end{array}$ & $\begin{array}{c}\text { Number of } \\
\text { Types of Ma- } \\
\text { jority Decision } \\
\text { Functions of } \\
n \text { Variables }\end{array}$ & $\begin{array}{c}\text { Number of Majority } \\
\text { Decision Functions of } \\
n \text { Variables }\end{array}$ & $\begin{array}{c}\text { Number of } \\
\text { Types of Self- } \\
\text { Dual Majority } \\
\text { Decision } \\
\text { Functions of } \\
n \text { Variables }\end{array}$ \\
\hline 1 & 4 & 1 & 1 & 2 & 1 \\
2 & 16 & 2 & 1 & 8 & 0 \\
3 & 10 & 3 & 72 & 1 \\
4 & 4,296 & 208 & 9 & 1,536 & 1 \\
5 & $4,967,296$ & 615,904 & 48 & 86,080 & 4 \\
6 & $18,446,774$, & - & 504 & $14,487,040$ & 14 \\
\hline
\end{tabular}

* These values are obtained from the results in References [4] and [5].

TABLE 3

The Maximum Values of Optimum Parameters of Majority Decision Functions

\begin{tabular}{c|c|c|c|c}
$n$ & $w$ & $V=\sum_{i=1}^{n} w_{i}$ & $T$ & $K$ \\
\hline 2 & 1 & 2 & 2 & 3 \\
3 & 2 & 4 & 3 & 5 \\
4 & 3 & 8 & 5 & 9 \\
5 & 5 & 16 & 9 & 17 \\
6 & 9 & 33 & 18 & 35 \\
\hline
\end{tabular}

variables the solution space of $(10)$ is a pointed cone. That is, there is a certain point $x_{0}$ such that

$$
A x_{0} \geqq b
$$

and any solution $x$ of (10) can be written as

$$
x=x_{0}+x^{\prime} \quad A x^{\prime} \geqq 0 .
$$

This means the solution space of (10) is a cone with $x_{0}$ as a sole vertex. These structures for majority decision functions of six variables were examined and it was found that almost all the majority decision functions have solution space of a pointed cone but that 15 out of 504 representatives have spaces of non-cone structure. These functions are marked with ${ }^{*}$ in Table 1.

Fourth, some maximum values of the optimum parameters are shown in Table 3 , where $V$ is the sum of coupling weights associated with input variables and $K$ is the total number of turns of windings including the constant winding and the relation $K=2 T-1$ holds. In Table 3,26 functions have the maximum value 9 for a weight $w$ and only one function attains the maximum value 33 of $V$; there are 7 functions with maximum $K$ of 35 . 
7. Acknowledgment. The authors wish to express their thanks to Mr. R. O. Winder, RCA Laboratories, Princeton, New Jersey, for his courtesy in comparing his data with ours, and to Dr. S. Takasu, Electrical Communication Laboratory, Tokyo, for his stimulating discussions.

International Business Machines Corporation

Thomas J. Watson Research Center

Yorktown Heights, New York

Electronics Research Section

Electrical Communication Laboratory

Musashino-shi, Tokyo, and

Electronics Research Section

Electrical Communication Laboratory

Musashino-shi, Tokyo

1. S. Muroga, I. Toda, S. Takasu, "Theory of majority decision elements," J. Franklin Inst., v. 271, n. 5, May 1961, p. 376-418.

2. I. Toda, M. Kondo, S. Muroga, "Majority decision functions of six variables," Electrical Communication Laboratory Technical Journal, v. 10, n. 3, 1961, p. 369-403, (in Japanese).

3. S. MURogA, "A computer program to find Boolean functions representable by a single logical element based on a majority decision principle," Electrical Communications Laboratory Technical Journal, v. 8, n. 6, 1959, p. 614-622, (in Japanese).

4. D. Slepian, "On the number of symmetry types of Boolean functions of $n$ variables," Canad. J. Math., v. 5, n. 2, 1953, p. 185-193.

5. B. Elspas, "Self-complementary types of Boolean functions," IRE Trans. on Electronic Computers, v. EC-9, n. 2, 1960, p. 264-266. 1960.

6. R. O. WINDER, "Single stage threshold logic," AIEE Conference Paper 60-1261, October

7. R. C. Minnick, "Linear-input logic," IRE Trans. on Electronic Computers, v. EC-10, n. 1, 1961, p. 6-16. 